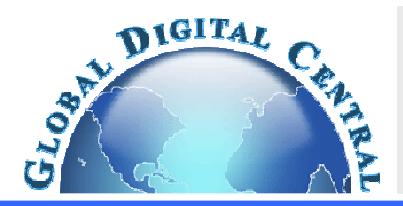

Frontiers in Heat and Mass Transfer

Available at www.ThermalFluidsCentral.org

\title{
HOT WATER COOLED HEAT SINKS FOR EFFICIENT DATA CENTER COOLING: TOWARDS ELECTRONIC COOLING WITH HIGH EXERGETIC UTILITY
}

\author{
Peter Kasten $^{\mathrm{a}}$, Severin Zimmermann ${ }^{\mathrm{a}}$, Manish K. Tiwari ${ }^{\mathrm{a}}$, Bruno Michel ${ }^{\mathrm{b}}$, Dimos Poulikakos ${ }^{\mathrm{a}, *}$ \\ ${ }^{a}$ Laboratory of Thermodynamics in Emerging Technologies, ETH Zurich, 8092 Zurich, Switzerland \\ ${ }^{\mathrm{b}}$ Advanced Thermal Packaging, IBM Zurich Research Laboratory, 8803 Rueschlikon, Switzerland
}

\begin{abstract}
Electronic data center cooling using hot water is proposed for high system exergetic utility. The proof-of-principle is provided by numerically modeling a manifold micro-channel heat sink for cooling microprocessors of a data center. An easily achievable $0.51 / \mathrm{min}$ per chip water flow, with $60^{\circ} \mathrm{C}$ inlet water temperature, is found sufficient to address the typical data center thermal loads. A maximum temperature difference of $\sim 8^{\circ} \mathrm{C}$ was found between the solid and liquid, confirming small exergetic destruction due to heat transport across a temperature differential. The high water outlet temperature from the heat sink opens the possibility of waste heat recovery applications.
\end{abstract}

Keywords: Hot water cooling, Exergy, data center, micro-channel manifold, heat sink

\section{INTRODUCTION}

State-of-the-art cooling systems cool the electronic components of data centers by forced air convection, wherein the cooling components can contribute up to the half of the total power consumption of data centers in order to chill and distribute the air (Brunschwiler et al. 2009, Koomey 2008). Further advances and miniaturization of electronic components have led and will lead to increased heat generation rates per unit area in electronic equipment, making the traditional cooling strategies insufficient (Brunschwiler and Michel 2008, Meijer 2010). Liquid cooling systems have the potential to overcome these obstacles due to the better thermophysical properties liquid compared to air and lower thermal resistance (by a factor of 5 or higher) of liquids cooling systems (Brunschwiler et al. 2006, Colgan et al. 2007). Electronic chip cooling using liquid has been explored extensively in the literature. Tuckerman and Pease(Tuckerman and Pease 1981) first investigated a liquid cooled micro-channel heat sinks and determined the internal thermal resistance of $0.09 \mathrm{~cm}^{2} \mathrm{~K} / \mathrm{W}$ for their best heat sink. The limitations of their approach are the high pressure drop and the nonuniform temperature distribution along the long parallel channels. As improvement, several studies proposed manifold micro-channel (MMC) heat sinks. In MMC heat sinks, the liquid coolant is supplied to and collected from the micro-channels (i.e. the heat transfer structure) in a direction normal to the base plate, supporting the channels, using supply manifolds. Copeland et al. (Copeland et al. 1997) developed a three dimensional and an analytical one dimensional model of a single micro-channel as a unit cell of the MMC heat sink. Their threedimensional model showed better agreement with their experimental data than their analytical one, which lacked accuracy for low flow rates. Fedorov and Viskanta (Fedorov and Viskanta 2000) used a finitedifference solving scheme for their three dimensional model and studied thoroughly the temperature and the heat flux distribution of a micro-channel unit cell. Ryu et al. (Ryu et al. 2003) also developed a three dimensional model of a MMC heat sink applying a finite-volume method and optimized the geometrical design parameters with the steepest descent technique. Their final design reduced the thermal resistance by $50 \%$ and improved the temperature variation compared to a micro-channel heat sink investigated in an earlier study. An interesting concept of self-contained oscillating flow based heat sink was explored by Waelchli et al. (Waelchli et al. 2010a, b). Escher and coworkers (Escher et al. 2010a, Escher et al. 2010b) were first to consider both the manifold and the micro-channel design in their investigations of an ultra-thin MMC heat sink. In order to minimize the computational cost, they approximated the micro-channels as a porous medium in a three dimensional hydrodynamic model of a manifold unit cell. They validated the pressure drop obtained through porous medium approach against their single micro-channel model. Based on the manifold model, they proposed and demonstrated a new manifold design providing uniform flow distribution over the heat transfer structure for improved heat transfer performance. Their optimized heat sink provides a thermal resistance of $0.087 \mathrm{~cm}^{2} \mathrm{~K} / \mathrm{W}$ and an overall pressure drop $<0.1$ bar.

The reduced thermal resistance in liquid cooling on one hand allows increased processor performance with the resulting high heat fluxes. On the other hand, use of liquid coolant can also reduce the thermal gradient needed for heat removal at low heat flux levels: For example, water cooling to remove the heat of today's products with power densities of $60 \mathrm{~W} / \mathrm{cm}^{2}$ only requires temperature gradients of $10^{\circ} \mathrm{C}$ as opposed to $60^{\circ} \mathrm{C}$ for air cooling solutions. Therefore, with water as coolant, the input coolant temperature can be raised above the hot season's free-cooling limit, thereby eliminating the need for coolant chillers in all climates, even on the hottest days. In addition, a high coolant temperature can also enable energy re-use, thereby improving the exergetic utilization of the system. For example, the hot liquid

*Corresponding author. Email: dimos.poulikakos@ethz.ch, Ph.: +41 44632 2738,Fax: +41 446321176 


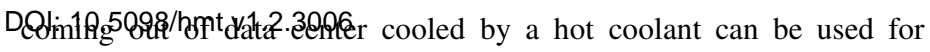
building heating. Such a holistic approach with energy re-use can minimize the overall carbon footprint at system level by reducing or eliminating the requirement of fossil fuels for building heating (Brunschwiler et al. 2009).

In this paper, we explore the concept of cooling electronics in a way that will enable the cooling of the data centers with "hot" water as a means to reduce carbon footprint of data centers and also enhance the exergetic utility of the cooling unit. We analyze a water cooled MMC heat sink, which is designed to cool the processors of a blade server and also explore the benefits of connecting the hot water at the outlet of blade server to a water heating system of the building for waste-heat recovery. First, a three dimensional conjugate heat transfer model of a micro-channel unit cell is developed to investigate the flow and the heat transfer characteristics of a chip simulator being cooled by hot water at $60^{\circ} \mathrm{C}$ inlet temperature. Furthermore, a three dimensional hydrodynamic model for the complete heat sink is also developed to study the flow distribution in the heat transfer structure as well as determining the overall pressure drop. The influence of flow distribution on thermal performance of heat sink is explained through a thermal resistance model. We follow a different approach than Escher at al. (Escher et al. $2010 \mathrm{~b}$ ), and model the hydrodynamics of both the inlet and the outlet manifolds and developed a turbulence model to capture the high shear stresses originating in heat sink, as explained further in the results and discussion section.

\section{GEOMETRY AND FABRICATION}

The investigated heat sink consists of the parallel micro-channel heat transfer structure and water distribution and collection manifolds as sketched in Figures 1 and 2. The heat sink design is similar to the one proposed by Escher et al. (Escher et al. 2010b), which was proven to be effective in removing high heat flux from processors in state-of-the-art datacenter blades. The water flow through the heat sink is marked with blue arrows in Figure 2. The coolant water is fed centrally to the inlet manifold with an inlet port connected to the hot water supply. The water from the inlet manifold flows through a slot nozzle in manifold's bottom wall on to the micro-channels and branches symmetrically into two parts to flow through the entire length of the channels. The nozzle slot design ensures that water impinges on the micro-channels enhancing heat transfer coefficient. The water leaves the microchannels at both ends via slot nozzles in the walls of collection manifolds and the two coolant streams recombine after a $90^{\circ}$ turn before leaving the manifold centrally. Geometrical parameters of the heat sink are given in Table 1.

Table 1: Geometric parameters of MMC heat sink

\begin{tabular}{|c|c|c|c|}
\hline Channel width, $w_{c h}[\mu \mathrm{m}]$ & 170 & $\begin{array}{l}\text { Outlet nozzle width, } \mathrm{w}_{\mathrm{n}, \text { out }} \\
{[\mathrm{mm}]}\end{array}$ & 2 \\
\hline Fin thickness, $\mathrm{w}_{\mathrm{fin}}[\mu \mathrm{m}]$ & 170 & $\begin{array}{l}\text { Inlet manifold width, } \\
\mathrm{w}_{\mathrm{M}, \text { in }}[\mathrm{mm}]\end{array}$ & 5 \\
\hline Channel height, $\mathrm{h}_{\mathrm{ch}}[\mathrm{mm}]$ & 1.7 & $\begin{array}{l}\text { Inlet manifold height, } \\
\mathrm{h}_{\mathrm{M}, \text { in }}[\mathrm{mm}]\end{array}$ & 4.25 \\
\hline Channel length, $\mathrm{L}_{\mathrm{ch}}[\mathrm{mm}]$ & 8.5 & $\begin{array}{l}\text { Outlet channel width }\left(1^{\text {st }}\right. \\
\text { leg }), w_{\mathrm{M}, \text { out }, 1}[\mathrm{~mm}]\end{array}$ & 5 \\
\hline Number of channels, $\mathrm{N}[-]$ & 62 & $\begin{array}{l}\text { Outlet channel width }\left(2^{\text {nd }}\right. \\
\text { leg }), w_{M, \text { out }, 2}[\mathrm{~mm}]\end{array}$ & 3 \\
\hline Base thickness, $\mathrm{h}_{\text {base }}[\mathrm{mm}]$ & 1.2 & $\begin{array}{l}\text { Outlet channel height, } \\
\mathrm{h}_{\mathrm{M} \text {,out }}[\mathrm{mm}]\end{array}$ & 4.5 \\
\hline $\begin{array}{l}\text { Inlet nozzle height, } \mathrm{h}_{\mathrm{n}, \text { in }} \\
{[\mathrm{mm}]}\end{array}$ & 1 & Inlet diameter, $\mathrm{d}_{\mathrm{M}, \mathrm{in}}[\mathrm{mm}]$ & 2.8 \\
\hline $\begin{array}{l}\text { Inlet nozzle width, } w_{n, \text {,in }} \\
{[\mathrm{mm}]}\end{array}$ & 2 & $\begin{array}{l}\begin{array}{l}\text { Outlet diameter, } \\
{[\mathrm{mm}]}\end{array} \\
\end{array}$ & 3 \\
\hline $\begin{array}{l}\text { Outlet nozzle height, } \mathrm{h}_{\mathrm{n}, \text { out }} \\
\text { [mm] }\end{array}$ & 0.75 & $\begin{array}{l}\text { Inlet manifold length, } \\
\mathrm{L}_{\mathrm{M}, \text { in }}[\mathrm{mm}]\end{array}$ & 21.25 \\
\hline
\end{tabular}

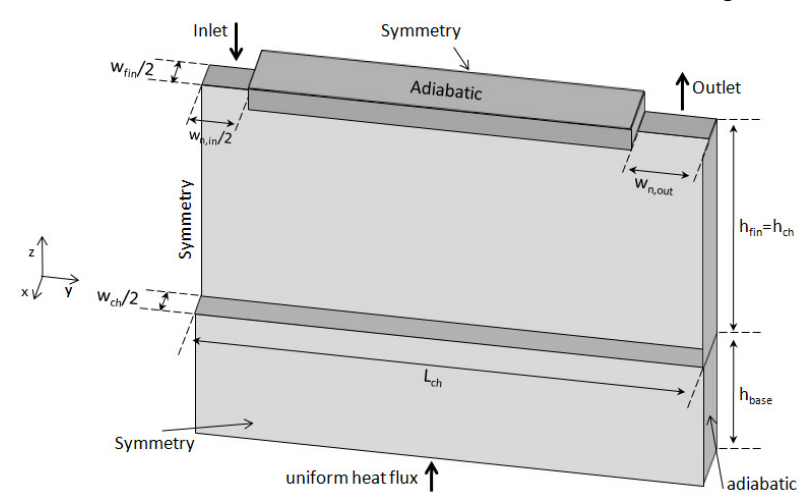

8629

Fig 1: Schematic of micro-channel unit cell domain

The investigated simplified design with only one inlet and two outlet nozzles is expected to cause a higher pressure drop, less heat removal and less uniform spatial temperature distribution within the cooled processor in comparison to MMC heat sinks with more sophisticated manifold designs (Escher et al. 2010b). The design is a compromise between fabrication cost and hydrodynamic and thermal performance which makes it well-suited for large-scale implementation in a prototype liquid cooled datacenter. The heat sink uses copper fins (micro channel walls) that are cut into the solid copper base plate and lifted using a patented Micro Deformation process (Wolverine Tube, Inc., USA), The inlet and outlet manifolds are machined into a second copper plate which is connected to the heat transfer structure by diffusion bonding. The dimensions of the entire heat sink are $47.5 \mathrm{~mm}$ $\mathrm{x} 47.5 \mathrm{~mm}$ in length and width. The dimensions of the heat sink fit the size of the copper cap mounted on top of processor packages in blade servers. This cap protects the processor from the surroundings and causes the necessary spatial temperature spreading for conventional aircooling. The aspect ratio of the fin is equal to 10 . Since the boundary layer is thinnest at the fin surface, the heat transfer through the fin is a major component of the heat removal from the entire heat sink.

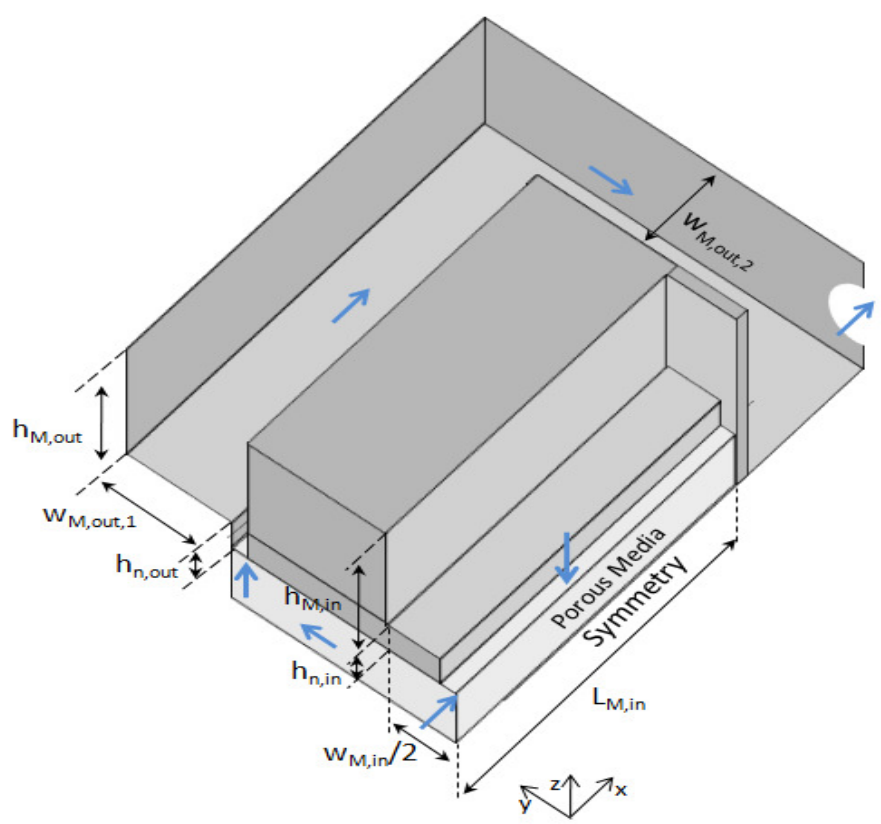

Fig 2: Schematic of manifold geometry (only half of the dissected along the symmetry plane is shown). 


\section{DOI: 10.5098/hmt.v13.30 \$UMERICAL MODELING}

\subsection{Micro-channel unit cell model}

The liquid is assumed as steady and incompressible and the thermophysical properties are all evaluated at the liquid inlet temperature and assumed to be constant. The continuity, the momentum and the energy equations for the liquid and energy equation for the solid parts are

$$
\begin{aligned}
& \frac{\partial u_{i}}{\partial x_{i}}=0 \\
& \rho u_{j} \frac{\partial u_{i}}{\partial x_{j}}=-\frac{\partial p}{\partial x_{i}}+\mu\left(\frac{\partial^{2} u_{i}}{\partial x_{j}^{2}}\right) \\
& \rho u_{i} \frac{\partial T_{f}}{\partial x_{i}}=\frac{k_{f}}{c_{p}} \frac{\partial^{2} T_{f}}{\partial x_{i}^{2}}+\frac{\mu}{c_{p}}\left(\frac{\partial u_{i}}{\partial x_{j}}+\frac{\partial u_{j}}{\partial x_{i}}\right) \frac{\partial u_{j}}{\partial x_{i}} \\
& \frac{\partial^{2} T_{s}}{\partial x_{i}^{2}}=0
\end{aligned}
$$

where the symbols $\rho, c_{p}, \mu, k, T, p$ and $u_{i}$ respectively denote the density, specific heat at constant pressure, dynamic viscosity, thermal conductivity, the temperature, the pressure and the velocity components. Table 2 lists the numerical values of the properties used.

\begin{tabular}{|l|l|}
\hline Density water, $\rho\left[\mathrm{kg} / \mathrm{m}^{3}\right]$ & 983.2 \\
\hline Specific heat capacity water, $c_{p}[\mathrm{~J} / \mathrm{kg} \mathrm{K}]$ & 4185 \\
\hline Dynamic viscosity water, $\mu[\mathrm{kg} / \mathrm{m} \mathrm{s}]$ & $4.6603 * 10^{-4}$ \\
\hline Thermal conductivity water, $k_{f}[\mathrm{~W} / \mathrm{m} \mathrm{K}]$ & 0.65439 \\
\hline Thermal conductivity copper, $k_{s}[\mathrm{~W} / \mathrm{m} \mathrm{K}]$ & 401 \\
\hline
\end{tabular}

Table 2: Thermo-physical properties used in the simulation

The solid and fluid domains in the model are coupled through the continuity of heat flux and temperature. Due to the periodicity of the parallel flow channel structure, we assume symmetry in the center of the channel and consider only half of the channel and half of the fin (the channel side wall) in the model. Symmetry is also imposed at the vertical plane where the flow enters and divides in two parts to fill the channel length. Assuming uniform flow distribution into all microchannels, the mass flow at the inlet to modeled channel-half and the channel Reynolds-number can be calculated by

$$
\begin{aligned}
& \dot{m}=\frac{\rho \dot{V}_{t o t}}{4 N}, \\
& R e_{c h}=\frac{\rho \bar{u}_{c h} D_{h, c h}}{\mu}=\frac{\rho \dot{V}_{t o t}}{N\left(w_{c h}+h_{c h}\right) \mu}
\end{aligned}
$$

where $N, \dot{V}_{t o t}, \bar{u}_{c h}, D_{h, c h}, w_{c h}$ and $h_{c h}$, respectively denote the number of micro-channels, the total volumetric flow rate, the density of water, the mean velocity in the micro-channel, the hydraulic diameter of the micro-channel, the micro-channel width and height, respectively. The no-slip condition is imposed at all walls and an opening condition of zero average static pressure is set at the outlet boundary allowing backflow into the domain. The later was validated by comparisons with separate simulation with an extended outlet channel. The top wall of the micro-channel is considered to be adiabatic as a big part of it is exposed to air while the dissipated heat of the processor is approximated as a uniform heat flux at the bottom wall. Due to large overhang of the micro-channels beyond the heat generating processor, the heat flux in the solid domain is directed primarily to near the inlet region of the micro-channel. Therefore, we neglect the heat transfer

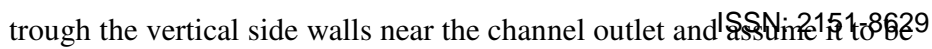
adiabatic.

The second order high resolution finite volumes scheme of the commercial Ansys CFX 12.1 package was used to solve Eqs. (1) - (4) applying the described boundary conditions while the convergence criterion was set to $10^{-6}$ for the root mean square values for all variables. An adequate block-structured, hexagonal, non-uniform grid of 901320 elements was used to obtain the results. Increasing the element number to 1761120 resulted in relative changes of $0.43 \%$, $0.050 \%$ and $0.106 \%$, respectively, in the pressure drop, the fluid temperature change from the inlet to the outlet, and the temperature difference between the maximal base plate and the fluid inlet temperatures.

\subsection{Thermal resistance model}

In addition to the detailed three dimensional-model of a single microchannel, a one dimensional thermal resistance model for a single microchannel is developed to estimate the thermal performance of the heat sink. The total thermal resistance separating the maximal heat sink temperature from the water inlet temperature can be divided in three parts. Firstly, since the heat is transferred by conduction through the base plate of the heat transfer structure, we define its thermal resistance as

$$
R_{\text {base }}=\frac{h_{\text {base }}}{\lambda_{s}}
$$

Secondly, convective heat transport from the solid to the water occurs through the fin surface and through the bottom and top wall of the micro-channels. Neglecting the heat transfer from the top wall to the liquid, we assume the thermal resistance of the fin and the bottom wall to act in parallel. Therefore, we defined the convective, thermal resistance as

$$
R_{\text {conv }}=\frac{w_{c h}+w_{f i n}}{w_{c h} h_{D}+2 h_{D} \eta_{f i n} h_{f i n}} .
$$

Spiga and Morini (Spiga and Morini 1996) analytically determined the Nusselt $(\mathrm{Nu})$ number for fully developed flow in rectangular ducts. Their value for a duct aspect ratio of 10 and constant heat flux from both long sides of the duct is $N u_{D}=6.096$. We used this to calculate the heat transfer coefficient for fully developed flow

$$
h_{D}=\frac{N u_{D} \lambda_{f}}{D_{h, c h}} .
$$

The analytical value of $\mathrm{Nu}$ from Spiga and Morini (Spiga and Morini 1996) can be used to estimate the heat transferred from the fin surfaces (side walls) and also to evaluate efficacy of fin surface in terms of heat transfer. First due to the high aspect ratio of the channels being used, it is important to ensure that the fin height is not too large, which could result in inefficient heat transport (i.e. the so called infinite fin length limit, see ref. (Incropera and DeWitt 2002)). The height of a fin in the infinite length limit can be evaluated as

$$
m h_{f i n, \infty}=4.6 \text {, with } m=\sqrt{\frac{2 h_{D}}{\lambda_{s} w_{f i n}}} .
$$

Equation (10) yields $h_{f i n, \infty}$ value of $7.5 \mathrm{~mm}$, which is well above the selected $1.7 \mathrm{~mm}$. Furthermore, assuming an insulated fin tip, the fin efficiency for can be computed as(Incropera and DeWitt 2002) 


$$
\begin{aligned}
& \frac{\partial}{\partial x_{j}}\left(\rho U_{j} k\right)=\frac{\partial}{\partial x_{j}}\left[\left(\mu+\frac{\mu_{t}}{\sigma_{k}}\right) \frac{\partial k}{\partial x_{j}}\right]+P_{k}-\beta^{\prime} \rho k \omega, \\
& \frac{\partial}{\partial x_{j}}\left(\rho U_{j} \omega\right)=\frac{\partial}{\partial x_{j}}\left[\left(\mu+\frac{\mu_{t}}{\sigma_{\omega}}\right) \frac{\partial \omega}{\partial x_{j}}\right]+\alpha \frac{\omega}{k} P_{k}-\beta \rho \omega^{2}, \\
& P_{k}=\mu_{t}\left(\frac{\partial U_{i}}{\partial x_{j}}+\frac{\partial U_{j}}{\partial x_{i}}\right) \frac{\partial U_{i}}{\partial x_{j}},
\end{aligned}
$$

ISSN: 2151-8629

Using Eq. (11), the fin efficiency is determined to be $75.0 \%$ for the considered thermo-physical properties and geometry. Note that Eq. (11) is derived under the assumption of a constant heat transfer coefficient from fin to fluid and constant fluid temperature, both of which are not at all true here (as will be clear from the Results and Discussion section). Therefore, efficiency values calculated here should just be used as an indication that we are not severely compromising the effectiveness of heat transport using too high pins (i.e. high channel aspect ratio).

Thirdly, the bulk resistance accounts for the heat transfer to the bulk flow and is visible in the temperature rise of the water. We defined it as

$$
R_{\text {bulk }}=\frac{L_{c h}\left(w_{c h}+w_{f i n}\right)}{c_{p} \rho^{\dot{V}_{t o t}} /(2 N)}
$$

By combining these three parts, the total thermal resistance can be expressed as

$$
R_{\text {tot }, 1 D}=R_{\text {base }}+R_{\text {conv }}+R_{\text {bulk }},
$$

since the three parts of the total resistance are connected in series.

\subsection{Hydrodynamic heat sink model}

The overall pressure drop of the heat sink and the flow distribution within the heat transfer structure are evaluated using a three dimensional model of the complete heat sink in which the parallel micro-channels are approximated as a porous medium in order to reduce computational load. Water enters centrally through a circular hole, connected to a hot water supply pipe, into the inlet manifold. Due to high flow rates (up to $11 / \mathrm{min}$ ), water travels rapidly to the back wall of the inlet manifold, which reverses it causing a backflow. The high shear stress at the interface between the forward and the backflow in the inlet manifold made it impossible to model the flow inside the manifold at steady state using the laminar model. Therefore, a turbulent flow model was used instead. A similar flow characteristic occurs in the second leg of the outlet manifold, where the two flow streams run into each other before leaving the manifold through outlet port connected to a pipe.

The Reynolds Averaged Navier-Stokes equations (RANS) were used to model the turbulent flow in the manifolds

$$
\begin{aligned}
& \frac{\partial U_{i}}{\partial x_{i}}=0 \\
& \rho U_{i} \frac{\partial U_{j}}{\partial x_{i}}=-\frac{\partial p}{\partial x_{j}}+\frac{\partial}{\partial x_{i}}\left[\left(\mu+\mu_{t}\right)\left(\frac{\partial U_{i}}{\partial x_{i}}+\frac{\partial U_{j}}{\partial x_{j}}\right)\right]+S_{p, i}, \\
& \mu_{t}=\rho \frac{k}{\omega} .
\end{aligned}
$$

The symbols $U_{i}, \mu_{t}, k, \omega$ and $S_{p, i}$ designate the mean velocity, eddy viscosity, turbulent kinetic energy, specific dissipation of kinetic energy and additional body force, respectively. The Wilcox' $k-\omega$ model (Wilcox 1998) equations, used for closure in the eddy viscosity approach, can be expressed as where the model constants are given as $\sigma_{k}=2, \sigma_{\omega}=2, \beta^{\prime}=0.09, \beta=$ 0.0075 and $\alpha=5 / 9$. An automatic wall treatment is used to capture the turbulence characteristics in the boundary layer, wherein a low Reynolds $k$ and $\omega$ formulation is blended into logarithmic wall function for $\mathrm{y}^{+}<11$, while pure logarithmic wall function approach is applied for $\mathrm{y}^{+}>11$. The wall boundary conditions used for $k$ and $\omega$ are:

$$
\begin{aligned}
& k=0, \\
& \omega=\frac{6 \mu}{\rho \beta(\Delta y)^{2}},
\end{aligned}
$$

where $\Delta y$ denotes the distance between the first two mesh points normal to the wall. Heyerichs and Pollard (Heyerichs and Pollard 1996) and Chen and Modi (Chen and Modi 1999) reported superior performance of the $k-\omega$ model over $k-\varepsilon$ models with enhanced wall treatment in recirculation flow situations. This is attributed to the more realistic treatment of the turbulence characteristics in the boundary layer and therefore was also used here.

The turbulent equations are also solved in the heat transfer structure approximated as porous medium although the flow in the microchannels is expected to be laminar. To test the influence of this on model performance, the section from the inlet to the outlet slot nozzles was simulated separately using the turbulent as well as laminar models. The effect of additional flow resistance due to the porous medium is found to be stronger than the turbulent effect (at least for the turbulence intensity levels observed in the entire heat sink simulation). The maximum relative difference between the turbulent and laminar models was $2.3 \%$ for uniform mass flow and turbulent intensity $I=0.4$ at the inlet boundary.

It is known from the literature that the $k-\omega$ model is strongly dependent on the inlet free stream boundary conditions of the turbulence equations (Wilcox 1998). Therefore, we varied the turbulence intensity and turbulence length scale at the manifold inlet in the range $0.005<I<0.06$ and $0.01 * d_{M, i n}<l<0.5 * d_{M, \text { in }}$, respectively. The overall pressure drop across the heat sink changed by $1.5 \%$ for the turbulence intensity variations and was even smaller for the variation of the turbulence length scale.

The additional momentum term $S_{p, i}$ in the Navier-Stokes equation accounts for the flow resistance of the porous medium and is set to zero in the inlet and the outlet manifolds. Since $\operatorname{Re}_{\mathrm{Ch}}>30$, the quadratic Forchheimer term is added to Darcy's law.

$$
S_{p, i}=-\frac{\mu}{\kappa_{i}} U_{i}-c_{l o s s} \kappa_{i}^{-0.5} \rho_{f}|U| U_{i}
$$

The constant loss coefficient $c_{\text {loss }}$ was evaluated by Beavers et al. (Beavers et al. 1973)as

$$
c_{\text {loss }}=0.55\left(1-5.5 \frac{D_{C h}}{D_{H T}}\right)
$$




$$
R_{t o t, 3 D}=\frac{T_{\text {max bottom }}-T_{f, \text { in }}}{q_{\text {chip }}^{\prime \prime}}
$$

the entire heat transfer structure, $D_{H T}$, as

$$
\begin{aligned}
& D_{C h}=\left(w_{C h} h_{C h}\right)^{0.5} \\
& D_{H T}=\left(L_{M, i n} h_{C h}\right)^{0.5} .
\end{aligned}
$$

Contrary to previous attempts to model the heat sink heat transfer structure as porous medium (Escher et al. 2010b, Jang and Kim 2005, Kim and Kim 1999), the permeability in the stream wise directions is assumed to be flow rate dependent. Equation (22) accounts for a straight flow through porous medium and does not take the two bends in the micro-channel, or the impingement on the surface or the interface between the fluid and the porous medium into account. Therefore, the stream wise permeability is determined by fitting the pressure drop from the inlet to the outlet nozzle to the pressure drop from the microchannel unit simulation while assuming the same boundary conditions as in the micro-channel model. A second order polynomial is found suitable to describe this flow dependent stream wise permeability as

$$
\kappa_{y, z} * 10^{9}=0.7715+5.101 * 10^{-4} \operatorname{Re}_{i n}+2.545 * 10^{-8} \operatorname{Re}_{i n}^{2},
$$

where all coefficients have a unit of $\mathrm{m}^{2}$. Since the micro-channel wall are impermeable, permeability in the $\mathrm{x}$ direction is taken to be up to 425 times smaller than the flow dependent values in Eq. (26). A progressively smaller reduction factors were used for lower flow rates, as a high value of reduction factor led to non-convergence of the model. This can be understood as follows. Equation (26) predicts that the permeability values in $\mathrm{y}$ and $\mathrm{z}$ directions increase monotonically with flow rate (i.e. $\mathrm{Re}_{\text {in }}$ ). Therefore, for small flow rates keeping the permeability reduction factor as high as 425 could lead to a very small value of permeability in $\mathrm{x}$ direction. Since permeability appears in the denominators of the Forchheimer terms in Eq. (22), an ultra low permeability will cause a strong spike in that term, which could lead to non-convergence.

A uniform velocity is imposed at the inlet port of the manifold and the average static pressure is set to zero at the outlet of an extended outlet pipe. The turbulence intensity $I$ and the turbulence length scale $l$ are assumed to be 0.02 and $0.07 * d_{M, \text { in }}$ at the inlet. Symmetry is used at the centerline of the heat sink and the no-slip condition is imposed at all walls.

The same solver and convergence criterion as in the micro-channel model were used to solve Eqs. (14) - (15) and a first order solver was used to solve the turbulence model Eqs. (17) - (18). We employed a block-structured, hexagonal, non-uniform grid of 3345328 elements in all simulations of the heat sink model as further grid refinement changed the overall pressure drop only by $0.058 \%$.

\section{RESULTS AND DISCUSSION}

In order to provide proof of principle for using the heat sink in a wasteheat recovery fluid loop, the inlet temperature was set to $T_{f, i n}=60^{\circ} \mathrm{C}$ in all simulations. The dissipated power of the chip is kept constant at $\dot{Q}_{\text {chip }}=90 \mathrm{~W}$ and the total volumetric flow rate is varied in the range 0.1 1/min $-11 /$ min. The inlet Reynolds-number defined as

$$
R e_{i n}=\frac{\rho \bar{u}_{i n} d_{M, i n}}{\mu}=\frac{\rho \dot{V}_{t o t}}{\mu \pi d_{M, i n} / 4},
$$

varies from $1599<R e_{i n}<15989$.

We evaluated the overall thermal and hydrodynamic performance of the heat sink considering the total thermal resistance and the pressure drop across the complete heat sink for varying volumetric flow rates (see Figure 3). The total thermal resistance of the heat sink decreases with increasing Reynolds-number in a power-law fashion as the convective heat transport to the fluid is enhanced by higher velocity gradients at the channel walls.

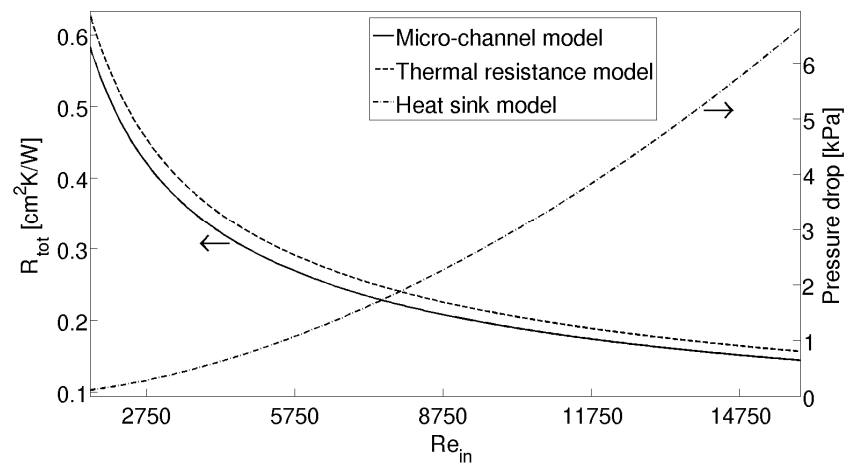

Fig 3: Overall performance of heat sink

\subsection{Micro-channel unit cell model}

The performance of the micro-channel model for different flow rates is first obtained in order to gain insight into the feasibility of momentum and energy transport inside the hot water cooled heat sink. To analyze the heat transfer in the micro-channel structure, the local Nusselt number, defined as

$$
N u_{y}=\frac{h_{y} D_{h, c h}}{k_{f}},
$$

is determined at 20 equally spaced locations along the channel. In Eq. (29), the local heat transfer coefficient is calculated using

$$
h_{y}=\frac{\bar{q}_{y, w}^{\prime \prime}}{\left(\bar{T}_{y, w}-\bar{T}_{y, b u l k}\right)},
$$

where $\bar{q}_{y, w}^{\prime \prime}, \bar{T}_{y, w}$ and $\bar{T}_{y, b u l k}$ respectively denote the average local wall heat flux density, the average local wall temperature and the local fluid bulk temperature defined, respectively as

$$
\begin{aligned}
& \bar{q}_{y, w}^{\prime \prime}=\left(\frac{1}{w_{c h}+h_{c h}}\right) * \\
& \left(\int_{0}^{w_{c h} / 2} \int_{0}^{q_{y, \text { ch, bottom }}^{\prime \prime}(x, 0) d x+} q_{y, \text { ch,top }}^{w_{c h} / 2}\left(x, h_{C h}\right) d x+\int_{0}^{h_{c h}} q_{y, \text { ch,fin }}^{\prime \prime}(0, z) d z\right), \\
& \bar{T}_{y, w}=\left(\frac{1}{w_{c h}+h_{c h}}\right) * \\
& \left(\int_{0}^{w_{c h} / 2^{T}} \int_{0}^{w_{c h} / 2} T_{y, \text { ch,top }}\left(x, h_{C h}\right) d x+\int_{0}^{h_{c h}} T_{y, \text { ch }, \text { fin }}(0, z) d z\right),
\end{aligned}
$$




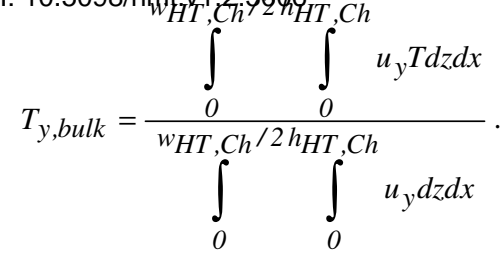

For analyzing the heat flux through the different micro-channel walls into water, a normalized heat flux density in the form

$$
\bar{q}_{y, i}^{*}=\frac{\bar{q}_{y, c h, i}^{\prime \prime}}{\bar{q}_{0.225 m m, c h, \text { fiin }}^{\prime \prime}}
$$

is introduced.

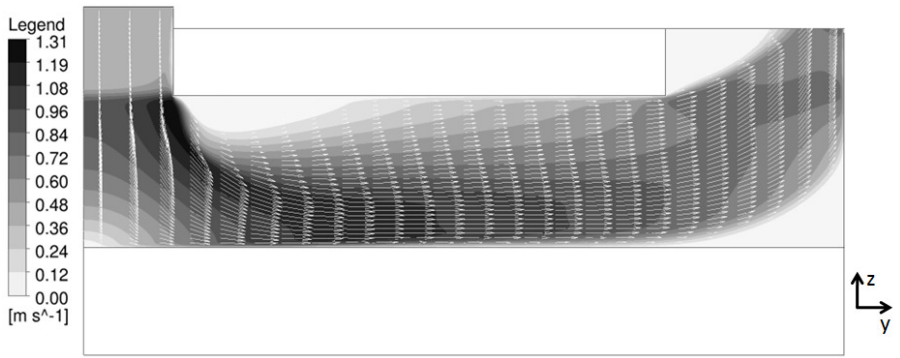

Fig 4: Vectors and velocity contours at center-plane of micro-channel model for $\mathrm{Re}_{\mathrm{Ch}}=303$ (volume flow rate $=1$ 1/min)

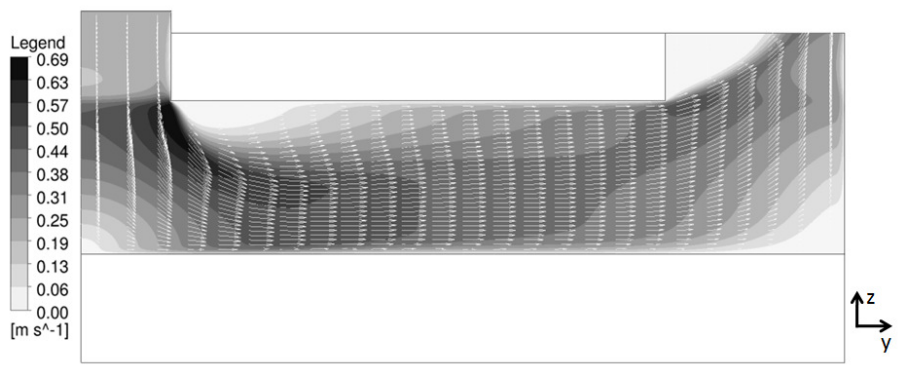

Fig 5: Vectors and velocity contours at center-plane of micro-channel model for $\mathrm{Re}_{\mathrm{Ch}}=152$ (volume flow rate $=0.5 \mathrm{l} / \mathrm{min}$ )

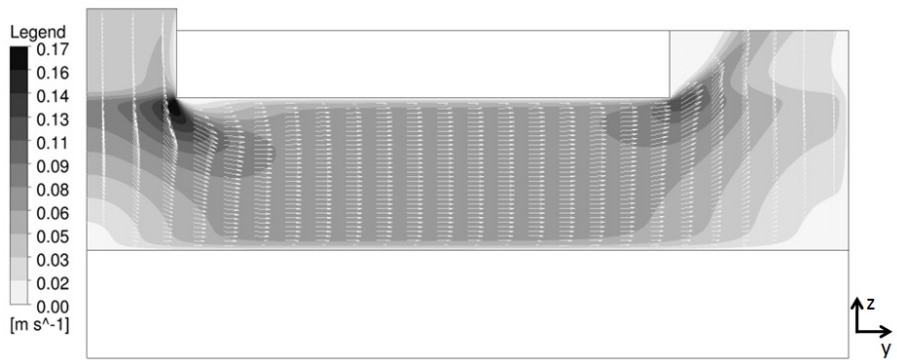

Fig 6: Vectors and velocity contours at center-plane of micro-channel model for $\mathrm{Re}_{\mathrm{Ch}}=30$ (volume flow rate=0.1 1/min)

The flow vectors and the velocity distribution at the centerline of the micro-channel for three different flow rates are presented in Figures $4-6$. The water enters the micro-channel by impinging onto the surface opposite to the inlet nozzle creating a small stagnation zone close to the vertical symmetry axis (Webb and Ma 1995). A recirculation zone is formed at the top wall near the inlet nozzle decreasing the effective flow cross section in the micro-channel and accelerating the flow. The size of this recirculation zone decreases with smaller flow rates and almost vanishes at the lowest investigated flow rate. Away from channel inlet, the liquid fills up the entire channel cross-section and the flow starts developing with respect to the top and bottom wall. In contrast, the flow is hydrodynamically fully developed with respect to the fin
Global Digital Central

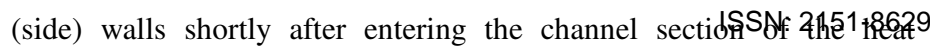
transfer structure. Near the outlet, a recirculation zone and a stagnation zone are being formed near the inner wall of the outlet nozzle and opposite to the outlet nozzle, respectively.

The differences in the flow field for varying flow rates create different temperature and heat transfer patterns along the microchannel. The average temperatures at the micro-channel outlet and at the heat sink bottom can be calculated as

$$
\begin{aligned}
& \bar{T}_{n, \text { out }}=\frac{1}{\left(w_{C h} / 2+w_{\text {fin }} / 2\right) w_{n, \text { out }}} * \\
& \int_{-w_{\text {fin }} / 2 L_{c h}-w_{n, \text { out }}}^{{ }^{w} C h / 2} \int_{\text {bottom }}=\frac{1}{\left(w_{C h} / 2+w_{\text {fin }} / 2\right) L_{c h}} \int_{-w_{\text {fin }} / 2}^{L_{C h} / 2} \int_{0}^{L_{c h}} T\left(z=-h_{\text {base }}\right) d y d x
\end{aligned}
$$

The temperature difference between the liquid inlet and the highest micro-channel temperatures is a measure of the gradients developing in the heat sink. These temperature differences are plotted in Figure 7 against $\operatorname{Re}_{\mathrm{Ch}}$ corresponding to the different flow rates considered in this work (up to $11 / \mathrm{min}$ ). High flow rates reduce the thermal resistance of the heat sink and cause a small fluid temperature change from the

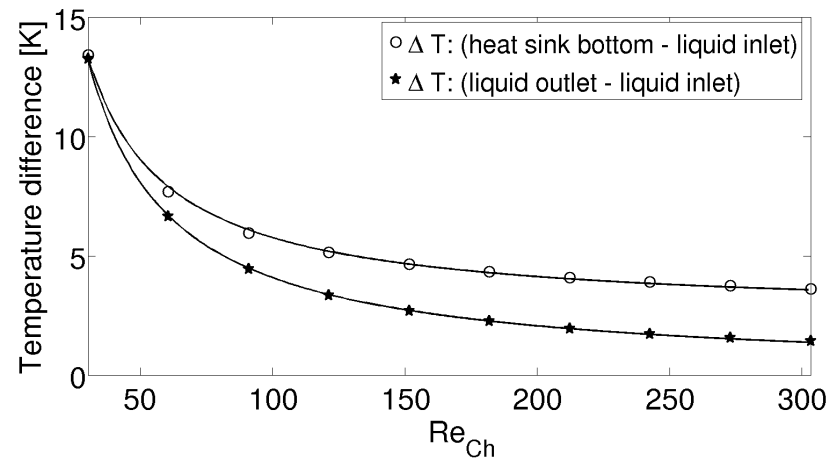

Fig 7: Difference of average heat sink bottom temperature and average fluid outlet temperature to fluid inlet temperature

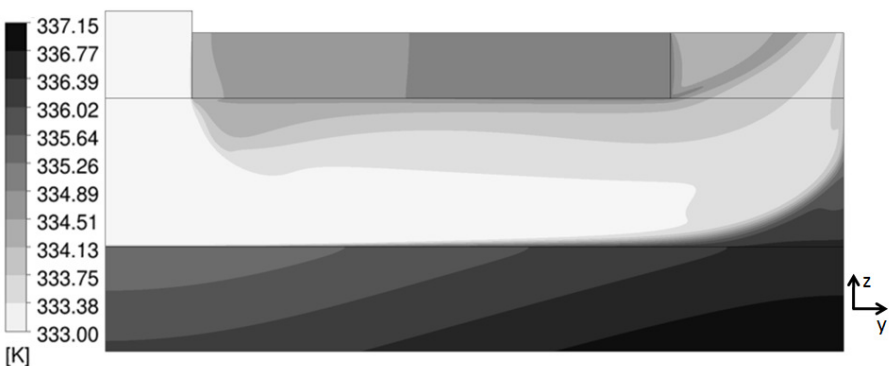

Fig 8: Temperature contours at center-plane of micro-channel model for $\mathrm{Re}_{\mathrm{Ch}}=303$ (volume flow rate=1 1/min)

micro-channel inlet to the outlet due to the short residence time within the channel. This effect is also visible in the temperature contours at the micro-channel center-plane which are shown for three different flow rates in Figures $8-10$. Areas of small temperature change coincide fairly well with the high flow velocity areas in Figures $4-6$. The highest temperatures are found in the downstream part of the heat sink bottom. The variations of the normalized heat flux density, through all the different solid-liquid interfaces in the micro-channel, along channel length are plotted in Figure 11. The trends in the heat flux density variation can be understood as follows. At high and medium flow rates, the water falls on to the channel bottom as a strong impinging jet. This jet impingement impedes the build-up of a hydrodynamic and a thermal 


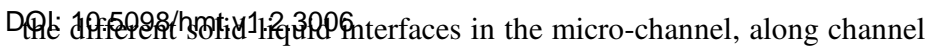
length are plotted in Figure 11. The trends in the heat flux density variation can be understood as follows. At high and medium flow rates, the different solid-liquid interfaces in the micro-channel, along channel

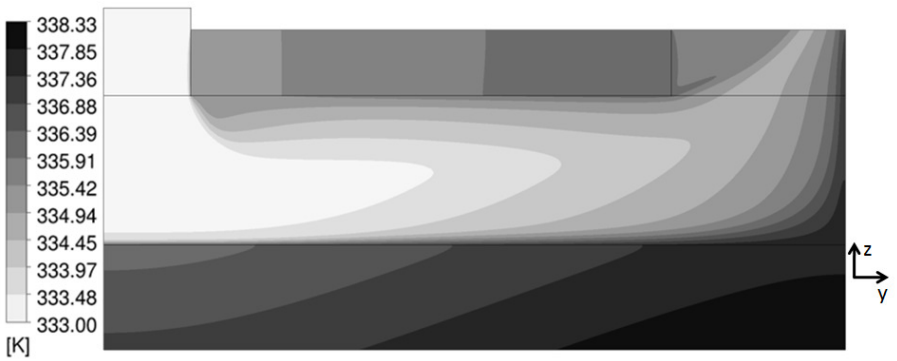

Fig 9: Temperature contours at center-plane of micro-channel model for $\mathrm{Re}_{\mathrm{Ch}}=152$ (volume flow rate $=0.5 \mathrm{l} / \mathrm{min}$ )

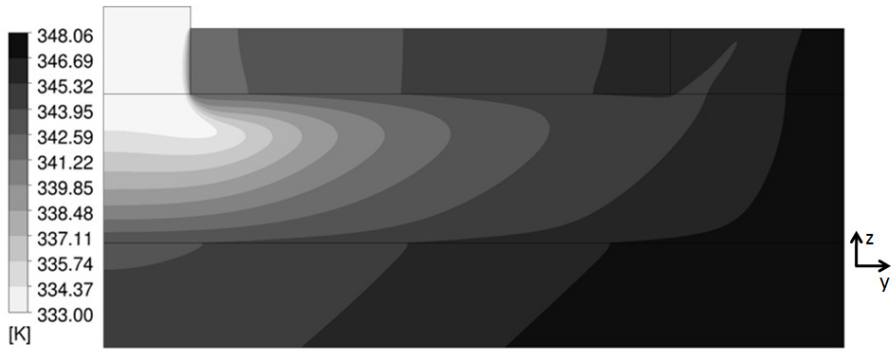

Fig 10: Temperature contours at center-plane of micro-channel model for $\mathrm{Re}_{\mathrm{Ch}}=30$ (volume flow rate $=0.1 \mathrm{1} / \mathrm{min}$ )

length are plotted in Figure 11. The trends in the heat flux density variation can be understood as follows. At high and medium flow rates, the water falls on to the channel bottom as a strong impinging jet. This jet impingement impedes the build-up of a hydrodynamic and a thermal boundary layer at the channel bottom reducing the thermal diffusion length to the convective fluid. Away from the inlet region, however, the thermal boundary layer growth results in a smaller heat transfer rate at the bottom channel (see Figure 11). In contrast, the inlet flow momentum is not high for low flow rate. Therefore, water does not impinge on the micro-channel bottom with sufficient strength and the heat flux is almost constant along the channel. The slight increase of the heat transfer rate in the first part of the channel is attributed to the increased velocity gradient in this section. For the fin (side) wall, the heat flux density is highest in the inlet region and decreases sharply downstream along the channel length. Right after the inlet region, the recirculation zone near the channel top reduces effective heat transfer area causing the heat transfer to decrease. Moreover, a thermal boundary layer should also start to grow on the fin surface and should

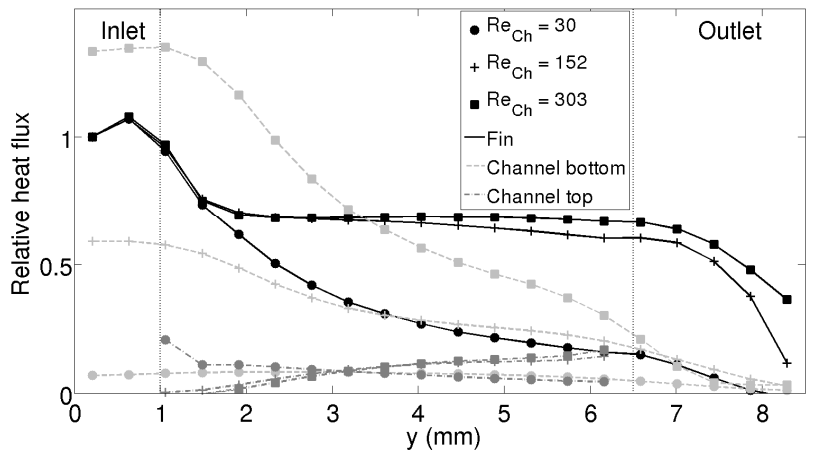

Fig 11: Axial development of relative heat flux density at channel bottom, channel top and fin wall

partially account for the heat flux reduction. Further downstream the liquid fills up the entire channel and the thermal boundary layer on the

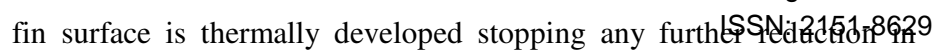
heat transfer. At low flow rates, contrary to high flow rates, the recirculation zone close to the inlet nozzle vanishes and the entire fin surface is effectively used for heat transfer. Therefore, the heat flux density through the fin decreases monotonically downstream due to the decreasing temperature gradient between fin surface and water bulk temperature. The variation of the recirculation zone near the top for different flow rates also affects the heat flux density at the top wall of the channel. At high and medium flow rates, the recirculation zone inhibits effective heat transfer trough the top wall close to the inlet nozzle. After the recirculation zone, the channel fills up resulting in increasing velocity gradient with respect to the top wall and therefore increasing heat flux density along the channel length. The vanishing recirculation zone allows for thermal boundary layer growth downstream causing the heat flux density to decrease. The stagnation zone opposite to the outlet nozzle causes a large diffusion length for heat transfer and a reduced heat flux density in this region. Since, in this region, the thermal resistance to the fin is smaller than to the fluid bulk, the heat is transported from the channel bottom through the liquid to the fin for small flow rates inducing the average heat flux to even turn negative and the bulk temperature to be higher than the average wall temperature.

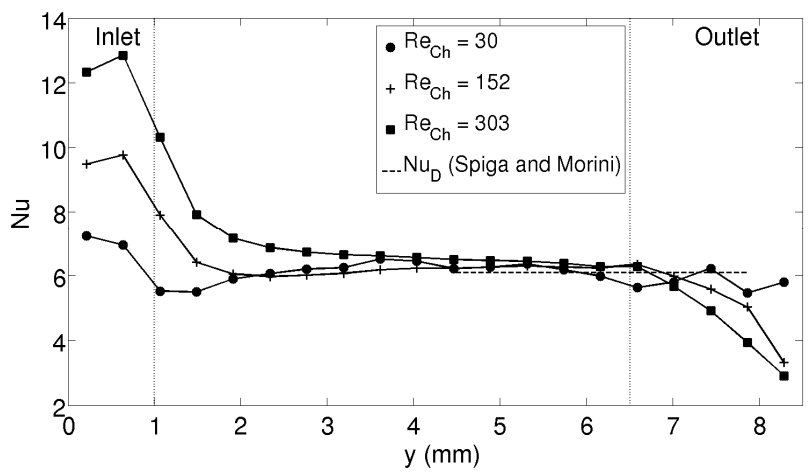

Fig 12: Axial development of Nu-number

The effect of progressively stronger impingement with increasing flow rate is evident in the axial development of the Nusselt number plotted in Figure 12, where it is higher for high volume flows in the inlet region of the micro-channel. Away from the inlet region and before the outlet, the flow becomes similar to a simple channel flow. The heat transfer through the fin surface is dominant in the investigated high aspect ratio micro-channel due to the large heat transfer area compared to the bottom and top wall of the channel. Moreover, the heat flux density of the fin surface is higher than for the other liquid-solid surfaces as the hydrodynamic and thermal boundary layers are thinnest for the fin surface. For all investigated flow rates, the flow is hydrodynamically and thermally fully developed for the fin surface shortly after entering the channel section causing the Nu-number to approach a value of $\approx 6.25$ close to end of the micro-channel, before the outlet section. Spiga and Morini (Spiga and Morini 1996) analytically determined Nusselt numbers for hydrodynamically and thermally fully developed laminar flow in rectangular ducts imposing constant heat flux to both long sides of the channel and considering the short sides to be adiabatic. Their value for channels of the aspect ratio 10 is $\mathrm{Nu}_{\mathrm{D}}=$ 6.096 , which serves as a good validation for the accuracy of the model developed here.

\subsection{Thermal resistance model}

The variation of the total thermal resistance of single micro-channel based on the thermal resistance model is presented Figure 3. The one dimensional resistance model overestimates the thermal resistance by less than $10 \%$ for all applied volumetric flow rates. The bulk resistance is the dominant thermal resistance and decreases with increasing flow rate. Therefore, the fluid temperature gradient within the micro-channel decreases with increasing flow rate. Since we assume an adiabatic tip of 
DQPL fipr 5998 (hm top wall to the fluid. Furthermore, we applied a constant heat transfer coefficient in our convective resistance term neglecting the enhanced heat transfer in the inlet region due to the impinging jet. These two assumptions may cause the thermal resistance model to under predict the heat transfer within the single micro-channel.

\subsection{Complete heat sink model}

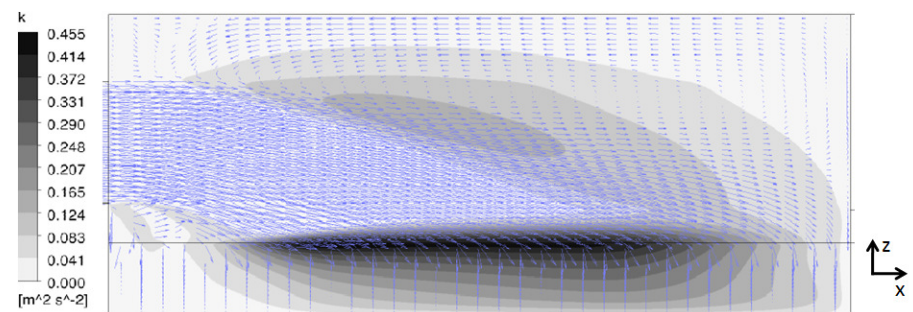

Fig 13: Turbulent kinetic energy contours and velocity vectors at center-plane of the inlet manifold at $\mathrm{Re}_{\text {in }}=7995$ (volume flow rate $=0.5$ $1 / \mathrm{min}$ )

In order to gain insight into the heat sink performance, it is also important to analyze the flow pattern in the manifold and the flow distribution within the heat transfer structure in addition to computing the overall pressure drop (Figure 3). The flow structure in the manifolds did not vary much at different flow rates considered here. Therefore, for the sake of brevity we only show the velocity vectors and the turbulence kinetic energy distributions for $\mathrm{Re}_{\mathrm{in}}=7995$ in Figure 13 and Figure 14. As seen from Figure 13, the coolant is laterally supplied through a pipe to the inlet manifold creating a flow core in the inlet manifold, which spreads slightly when approaching towards the heat transfer structure.

A small vortex is generated close to the inlet due to the backwardfacing step type geometry. Additionally, the back wall of the inlet manifold, at the far end in the flow direction in Figure 13, induces a large recirculation zone above the inlet flow core. The flow core drags the surrounding fluid in the flow direction generating high shear stresses and turbulence which is shown by the high turbulence kinetic energy in this area. Figure 14 shows the velocity vectors and turbulent kinetic energy contours for the mid yz-plane of the heat sink. It clarifies the flow patterns in the heat transfer structure modeled as porous medium. Upon entering the porous medium, the liquid momentum in flow direction changes sharply, creating a second zone of high turbulence. However, the turbulence kinetic energy dissipates quickly while flowing through the porous medium and remains small in the first leg of the outlet section of the manifold (see Figure 14). The quick turbulence dissipation in the first part of the porous medium (heat transfer structure) shows that turbulent modeling of the hydrodynamics of entire heat sink is not compromised by low turbulence in heat transfer structure (micro-channels).Therefore, use of a laminar model (as was done in Section 4.1) is adequate to evaluate the heat transfer in single micro-channel unit cell. A detailed thermal model for entire heat sink including the inlet and outlet manifolds, however, should certainly use turbulent approach. This will be considered in our future work.

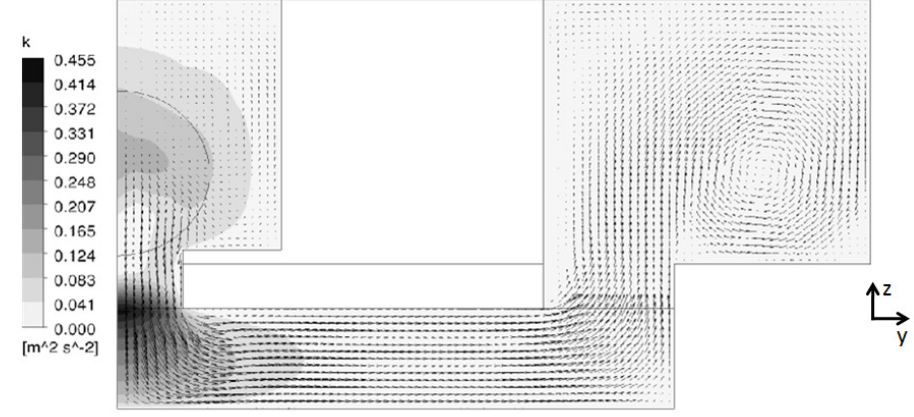

Fig 14: Turbulent kinetic energy contours and velocity vectors of yzplane at $\mathrm{x}=\mathrm{L}_{\mathrm{M}, \mathrm{in} / 2}$ for $\mathrm{Re}_{\mathrm{in}}=7995$ (volume flow rate $=0.5 \mathrm{l} / \mathrm{min}$ )

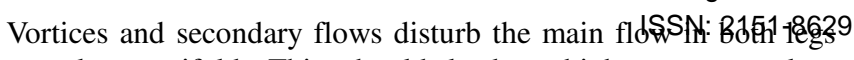
of the outlet manifold. This should lead to higher pressure loss especially at high flow rates. The flow streams from two parts of the first leg of the outlet manifold run into each other in the second leg. This generates turbulence kinetic energy and high shear stresses contributing further to the pressure loss.

Distribution of coolant in the micro-channels is crucial to ensure improved performance of heat sink. In order to extract the mass flux distribution into the channels, we divided the cross section of the heat transfer structure at $\mathrm{y}=\mathrm{L}_{\mathrm{Ch}} / 2$ in equally spaced sections of width $\Delta x$. The relative mass flow rate was calculated using

$$
\dot{m}_{i}^{*}=\frac{\int_{x_{i}-\Delta x / 2}^{x_{i}+\Delta x / 2} \int_{0}^{h_{H T} C h} \dot{m}_{i} d z d x}{\frac{1}{N} \sum_{i=1}^{N} \int_{x_{i}-\Delta x / 2}^{x_{i}+\Delta x / 2} \int_{0}^{h} \dot{m}_{i} d z d x} .
$$

The resulting relative mass fluxes are plotted in Figure 15 for the three different flow rates designated by three different $\mathrm{Re}_{\text {in }}$. The mass flux distribution into the micro-channels seems to vary quite strongly depending on the channel position in $\mathrm{x}$ direction. This is expected since the water enters as a jet into the inlet manifold (c.f. Figure 13). The three different flow rates in Figure 15 correspond to $\mathrm{Re}_{\mathrm{Ch}}$ values of 30 , 152 and 303 respectively, if the flow distribution into all channels were to be uniform. Due to non-uniform flow distribution, the lowest relative mass fractions into channels, as seen from Figure 15, are 0.35, 0.4 and 0.55 respectively, for the low $(0.1 \mathrm{l} / \mathrm{min})$, medium $(0.5 \mathrm{l} / \mathrm{min})$ and high (1 $1 / \mathrm{min}$ ) flow rates considered. These fractions correspond to lowest $\operatorname{Re}_{\mathrm{Ch}}$ values of 10.5, 61 and 167, respectively. A low value of $\mathrm{Re}_{\mathrm{Ch}}$ can lead to an unacceptably high chip temperature as seen in Figure 7. Moreover, the minimum $\operatorname{Re}_{\mathrm{Ch}}$ studied, i.e. $\mathrm{Re}_{\mathrm{Ch}}=30$ (with corresponding volumetric flow rate of $0.1 \mathrm{l} / \mathrm{min}$ ), leads to a $15^{\circ} \mathrm{C}$ difference between maximum heat sink and water inlet $\left(60^{\circ} \mathrm{C}\right)$ temperatures. Therefore, the minimum $\mathrm{Re}_{\mathrm{Ch}}$ of 10.5 observed for the lowest flow rates will clearly lead to a much higher chip temperature, which makes the lowest flow rate unacceptable. However, for the medium and high flow rates, the minimum $\operatorname{Re}_{\mathrm{Ch}}$ values (61 and 167) are high enough for a very good thermal performance of heat sink with inlet water temperature of $60^{\circ} \mathrm{C}$. The maximal temperature difference of $\sim 8^{\circ} \mathrm{C}$ (see Figure 7) for these flow rates should ensure a minimal entropy generation due to a heat transport across a temperature differential. The higher mass flows through the micro-channels lying in the middle of the heat sink (see Figure 15) should only improve the cooling effectiveness and not degrade it. Moreover, the outlet temperature of at least $62^{\circ} \mathrm{C}$, makes it useful for secondary applications such as heating the building hosting such as hot water cooled datacenter. Experimental validation of this principle and investigation into a prototype water cooled datacenter is currently under investigation by group and would be reported in near future.

\section{CONCLUSION AND OUTLOOK}

Using numerical modeling, feasibility of electronic datacenter cooling using hot water is explored for high system exergetic utility. The model considers a manifold micro-channel (MMC) heat sink, with high aspect ratio (10) micro-channels, suitable for large scale fabrication and integration. A three dimensional model of conjugate heat transport in a micro-channel unit cell, a thermal resistance and a three dimensional hydrodynamic model of the complete heat sink were developed to evaluate the thermal performance of the heat sink and also to explore the possibility of waste-heat recovery. In the heat sink hydrodynamic model, a $k-\omega$ type turbulent model was used to capture the high shear stresses in the supply and collection manifolds, whereas the flow 


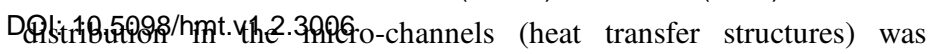
obtained by modeling the heat transfer structure as porous medium, which saved computation time.

As Expected, the thermal resistance of the heat sink decreased with increasing flow rate while the pressure drop across the heat sink increased. The local Nu-number close to the outlet of the high aspect ratio micro-channels approached 6.25 , a value comparing well with the literature value of 6.096 for channels heated through two walls. The manifold was found to distribute the flow non-uniformly to the microchannels. The repercussion of this non-uniform distribution was analyzed. Indeed, an easily achievable minimum flow rate of $0.51 / \mathrm{min}$ for water at $60^{\circ} \mathrm{C}$ inlet temperature was found sufficient to address the thermal loads typical in a datacenter chip. The maximum temperature difference of $\sim 8^{\circ} \mathrm{C}$ was found between the solid and liquid, confirming small exergetic destruction due to heat transport across a temperature differential. Furthermore, the high water outlet temperature from the heat sink opens the possibility for secondary use of the waste heat.

A conjugate heat transport model of the entire heat sink and experimental validation of the proof-of-principle of hot water cooled datacenters will be investigated in our future work.

\section{ACKNOWLEDGEMENTS}

We acknowledge G. I. Meijer and S. Paredes for sharing the mechanical design parameters (Table 1) and thermal simulations of these cold plates, which are used in the IBM BladeCenter Cluster Aquasar.

\section{NOMENCLATURE}

\begin{tabular}{|c|c|}
\hline$c_{p}$ & specific heat capacity $(\mathrm{J} / \mathrm{kg} \cdot \mathrm{K})$ \\
\hline$D$ & characteristic length scale (m) \\
\hline$D_{h}$ & Hydraulic diameter (m) \\
\hline$d$ & diameter $(\mathrm{m})$ \\
\hline$h$ & height (m) \\
\hline$h$ & heat transfer coefficient $\left(\mathrm{W} / \mathrm{m}^{2} \cdot \mathrm{K}\right)$ \\
\hline$k$ & turbulent kinetic energy $\left(\mathrm{m}^{2} / \mathrm{s}^{2}\right)$ \\
\hline$k$ & thermal conductivity $(\mathrm{W} / \mathrm{m} \cdot \mathrm{K})$ \\
\hline$L$ & length (m) \\
\hline$\dot{m}$ & mass flow rate $(\mathrm{kg} / \mathrm{s})$ \\
\hline$\dot{m}^{*}$ & relative mass flow rate $(-)$ \\
\hline$N$ & number (-) \\
\hline $\mathrm{Nu}$ & Nusselt-number (-) \\
\hline$p$ & pressure $\left(\mathrm{N} / \mathrm{m}^{2}\right)$ \\
\hline$q "$ & heat flux density $\left(\mathrm{W} / \mathrm{m}^{2}\right)$ \\
\hline $\mathrm{q}^{*}$ & relative heat flux density (-) \\
\hline$R$ & thermal resistance $\left(\mathrm{m}^{2} \cdot \mathrm{K} / \mathrm{W}\right)$ \\
\hline$R e$ & Reynolds-number (-) \\
\hline$S_{p}$ & body force due to porous medium $\left(\mathrm{kg} / \mathrm{m}^{2} \cdot \mathrm{s}^{2}\right)$ \\
\hline$T$ & temperature $(\mathrm{K})$ \\
\hline$U$ & mean velocity $(\mathrm{m} / \mathrm{s})$ \\
\hline$u$ & velocity $(\mathrm{m} / \mathrm{s})$ \\
\hline$\dot{V}$ & volumetric flow rate $\left(\mathrm{m}^{3} / \mathrm{s}\right)$ \\
\hline$w$ & width (m) \\
\hline$x_{i}$ & coordinates $(\mathrm{m})$ \\
\hline$y^{+}$ & dimensionless wall distance (-) \\
\hline$\Delta y$ & distance between first mesh points normal to the wall (m) \\
\hline \multicolumn{2}{|c|}{ Greek Symbols } \\
\hline$\alpha$ & closure coefficient of k- $\omega$ model (-) \\
\hline$\beta$ & closure coefficient of k- $\omega$ model (-) \\
\hline$\beta$ & closure coefficient of $k-\omega$ model $(-)$ \\
\hline$\eta$ & fin efficiency (-) \\
\hline$\lambda$ & thermal conductivity $(\mathrm{W} / \mathrm{m} \cdot \mathrm{K})$ \\
\hline$\mu$ & dynamic viscosity $(\mathrm{kg} / \mathrm{m} \cdot \mathrm{s})$ \\
\hline$\mu_{t}$ & eddy viscosity $(\mathrm{kg} / \mathrm{m} \cdot \mathrm{s})$ \\
\hline$\rho$ & density $\left(\mathrm{kg} / \mathrm{m}^{3}\right)$ \\
\hline$\sigma_{k}$ & closure coefficient of k- $\omega$ model (-) \\
\hline$\sigma_{\omega}$ & closure coefficient of $\mathrm{k}-\omega$ model (-) \\
\hline
\end{tabular}

$\omega$ Subscripts

base heat sink base plate

bottom bottom of micro-channel or heat sink

bulk bulk

ch micro-channel

conv convection

D developed

$f \quad$ fluid

fin fin

HT heat transfer structure

$i \quad$ index

in inlet

M manifold

$n \quad$ nozzle

out outlet

$s \quad$ solid material

tot total

w wall

\section{REFERENCES}

Beavers, G. S., Sparrow, E. M., and D.E., R., 1973, "Influence of Bed Size on the Flow Characteristics and Porosity of Randomly Packed Beds of Spheres," Transactions ASME, Series E, Journal of Applied Mechanics, 40 (3), 655-660.

Brunschwiler, T., and Michel, B., 2008, "Thermal Management of Vertically Integrated Packages," Handbook of 3D Integration, WILEYVCH Verlag GmbH \& Co, KGaA, Weinheim.

doi: 10.1002/9783527623051.ch33

Brunschwiler, T., Rothuizen, H., Fabbri, M., Kloter, U., Michel, B., Bezama, R. J., and Natarajan, G., 2006, "Direct Liquid Jet-Impingement Cooling with Micron-Sized Nozzle Array and Distributed Return Architecture," IEEE Proceedings 10th Intersociety Conference on Thermal and Thermomechanical Phenomena in Electronics Systems. doi: 10.1109/ITHERM.2006.1645343

Brunschwiler, T., Smith, B., Ruetsche, E., and Michel, B., 2009, "Toward Zero-Emission Data Centers through Direct Reuse of Thermal Energy," IBM Journal of Research \& Devlopement, 53 (3), Paper 11. doi: 10.1147/JRD.2009.5429024

Chen, Q., and Modi, V., 1999, "Mass Transfer in Turbulent Impinging Slot Jets," International Journal of Heat and Mass Transfer, 42 (5), 873-887.

doi: 10.1016/S0017-9310(98)00215-4

Colgan, E. G., Furman, B., Gaynes, M., Graham, W. S., Labianca, N. C., Magerlein, J. H., Polastre, R. J., Rothwell, M. B., Bezama, R. J., Choudhary, R., Marston, K. C., Toy, H., Wakil, J., Zitz, J. A., and Schmidt, R. R., 2007, "A Practical Implementation of Silicon Microchannel Coolers for High Power Chips," IEEE Transactions on Components and Packaging Technologies, 30 (2), 218-225. doi: 10.1109/tcapt.2007.897977

Copeland, D., Behnia, M., and Nakayama, W., 1997, "Manifold Microchannel Heat Sinks: Isothermal Analysis," IEEE Transactions on Components, Packaging, and Manufacturing Technology Part A, 20 (2), $96-102$.

doi: $10.1109 / 95.588554$

Escher, W., Brunschwiler, T., Michel, B., and Poulikakos, D., 2010a, "Experimental Investigation of an Ultra-Thin Manifold Microchannel Heat Sink for Liquid-Cooled Chips," ASME Journal of Heat Transfer, 132 (8), 081402.

doi: $10.1115 / 1.4001306$ 
Frontiers in Heat and Mass Transfer (FHMT), 1, 023006 (2010)

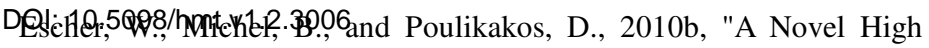
Performance, Ultra Thin Heat Sink for Electronics," International Journal of Heat and Fluid Flow, 31 (4), 586-598.

doi: 10.1016/j.ijheatfluidflow.2010.03.001

Fedorov, A. G., and Viskanta, R., 2000, "Three-Dimensional Conjugate Heat Transfer in the Microchannel Heat Sink for Electronic Packaging," International Journal of Heat and Mass Transfer, 43 (3), 399 - 415. doi: 10.1016/S0017-9310(99)00151-9

Heyerichs, K., and Pollard, A., 1996, "Heat Transfer in Separated and Impinging Turbulent Flows," International Journal of Heat and Mass Transfer, 39 (12), 2385-2400.

doi: $10.1016 / 0017-9310(95) 00347-9$

Incropera, F. P., and Dewitt, D. P., 2002, Fundamentals of Heat and Mass Transfer, $5^{\text {th }}$ ed., John Wiley \& Sons, Inc.

Jang, S. P., and Kim, S. J., 2005, "Fluid Flow and Thermal Characteristics of a Microchannel Heat Sink Subject to an Impinging Air Jet," Journal of Heat Transfer-Transactions of the ASME, 127 (7), 770-779.

doi: $10.1115 / 1.1924628$

Kim, S. J., and Kim, D., 1999, "Forced Convection in Microstructures for Electronic Equipment Cooling," Journal of Heat TransferTransactions of the ASME, 121 (3), 639-645.

doi: $10.1115 / 1.2826027$

Koomey, J. G., 2008, "Worldwide Electricity Used in Data Centers," Environmental Research Letters, 3 (3).

doi: 10.1088/1748-9326/3/3/034008

Meijer, G. I., 2010, "Cooling Energy-Hungry Data Centers," Science, 328 (5976), 318-319.

doi: $10.1126 /$ science. 1182769
Global Digital Central

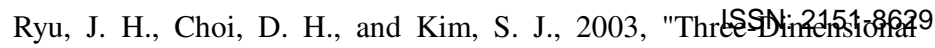
Numerical Optimization of a Manifold Microchannel Heat Sink," International Journal of Heat and Mass Transfer, 46 (9), 1553 - 1562. doi: 10.1016/S0017-9310(02)00443-X

Spiga, M., and Morini, G. L., 1996, "Nusselt numbers in laminar flow for H2 boundary conditions," International Journal of Heat and Mass Transfer, 39 (6), 1165-1174. doi: 10.1016/0017-9310(95)00205-7

Tuckerman, D. B., and Pease, R. F. W., 1981, "High-performance heat sinking for VLSI," Electron Device Letters, 2 (5), 126-129.

Waelchli, R., Brunschwiler, T., Michel, B., and Poulikakos, D., 2010a, "Combined local microchannel-scale CFD modeling and global chip scale network modeling for electronics cooling design," International Journal of Heat and Mass Transfer, 53 (5-6), 1004-1014. doi: 10.1016/j.ijheatmasstransfer.2009.11.012

Waelchli, R., Brunschwiler, T., Michel, B., and Poulikakos, D., 2010b, "Self-Contained, Oscillating Flow Liquid Cooling System for Thin Form Factor High Performance Electronics," Journal of Heat TransferTransactions of the Asme, 132 (5). doi: 051401 10.1115/1.4000456

Webb, B. W., and Ma, C. F., 1995, "Single-phase liquid jet impingement heat transfer," Advances in Heat Transfer, 26, 105-217. doi: 10.1016/S0065-2717(08)70296-X

Wilcox, D. C., 1998, Turbulence Modeling for CFD, $2^{\text {nd }}$ ed., DWC Industries, La Canada, California. 